\title{
The rough entropy of multi-granularity rough set to interval number information system
}

\author{
Yu YingYing ${ }^{1,}$, , Hu Qian ${ }^{2, b}$
}
${ }^{1}$ construction technical college of guangxi, The ministry of public foundation,Nanning Guangxi China ,530002

${ }^{2}$ Changjiang University School of Information and Mathematics, Wuhan Hubei China ,430010

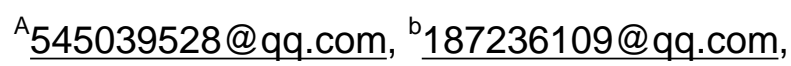

Keywords: Multi-granularity rough set ; dominance relation; rough entropy

Abstract: In interval-valued information system, the rough entropy to Multi granularity rough set is presented, the quality of rough set is described by dominance relation, Condition of rough entropy is raised to discuss the nature, the example is used to analysis specific.

\section{Introduction}

In 1948,Shannon takes into account that the entropy of measurement of electrical, mechanical, and thermal systems has unordered and disordered defects, Entropy is proposed to measure the uncertainty of various systems, And the Shannon entropy (information entropy), Many scholars in rough sets have also used entropy to analyze the fuzziness and uncertainty of information system in the advantage relation.

Many scholars consider the advantages of entropy in the information system [2]

.The rough entropy of the rough set of probability in the advantage relation is analyzed by the probability introduction by wang guying [3], The correlation between knowledge granularity and rough entropy is analyzed in the rough entropy with the probability of parameter.GUI XIAN CAI[4] proposed the extended conditional entropy and used the rough decision entropy to measure the stability of the interval number information system.

However, there are some shortcomings in the study of single-particle space in rough sets,For example, in part of the decision question,If two decision-makers are independent of each other, then any two knowledge mixing operations cannot be operable.In order to deal with the lack of partition in single particle size space,Qian yuhua [5] first proposed the multi-granularity information system in 2006, using the equivalence relation to divide the information system.Li qi fang [6] discussed some of the properties of the coarse-grained rough set in the advantage relation;Zhai yongjian [7] introduced the dominant relationship to the multi-particle coarse fuzzy set and its corresponding incomplete information system.

In order to further study the rough entropy of the multi-granularity rough set in the interval value information system, in this paper, the properties of rough set model are described by using the advantages relation, and the properties of the rough set model are presented by the rough entropy, and the example is used to analyze the model.

basic concept

interval number information system

An information system $I S=<U, A T, V, f>, U$ is domain of discourse, $A T=A \cup\{d\}$ is set of properties, $A$ is Conditional attribute set, $d$ is Decision attribute; $\forall a \in A, U \rightarrow V_{a}$ Among $V_{a}$ is set of properties, $V$ is the corresponding attribute range; $V=V_{A T}=\underset{a \in A T}{U} V_{a}, \forall x \in U, f(x, a)$ Represents the value of an object $x$ under attribute $a \in A T ; f(x, a)=\left[a^{L}(x), a^{U}(x)\right]_{\text {expression to represent the }}$ interval number under the property $a$, and $a^{L}(x) \leq a^{U}(x)$. 
Dominance relation

An information system $I S=<U, A T, V, f>$, $\forall x_{i}, x_{j} \in U, a \in A, f\left(a, x_{i}\right)=\left[a^{L}\left(x_{i}\right), a^{U}\left(x_{i}\right)\right],|\cdot|$ expression length of interval,the degree $p_{i j}^{a}$ of $x_{i}$ better than ${ }^{x_{j}}$ is divided into the following situations:

(1) $a^{L}\left(x_{j}\right) \leq a^{U}\left(x_{j}\right) \leq a^{L}\left(x_{i}\right) \leq a^{U}\left(x_{i}\right)$, $p_{i j}^{a}=1$;

(2) $a^{L}\left(x_{j}\right) \leq a^{L}\left(x_{i}\right)<a^{U}\left(x_{j}\right) \leq a^{U}\left(x_{i}\right)$, $p_{i j}^{a}=\frac{\frac{1}{2}\left[a^{U}\left(x_{j}\right)-a^{L}\left(x_{i}\right)\right]}{\left|f\left(a, x_{i}\right) \cup f\left(a, x_{j}\right)\right|}+\frac{a^{U}\left(x_{i}\right)-a^{U}\left(x_{j}\right)+a^{L}\left(x_{i}\right)-a^{L}\left(x_{j}\right)}{\left|f\left(a, x_{i}\right) \cup f\left(a, x_{j}\right)\right|}$

(3) $a^{L}\left(x_{i}\right)<a^{L}\left(x_{j}\right)<a^{U}\left(x_{j}\right) \leq a^{U}\left(x_{i}\right)$, $p_{i j}^{a}=\frac{\left[a^{U}\left(x_{i}\right)-a^{U}\left(x_{j}\right)\right]}{\left|f\left(a, x_{i}\right) \cup f\left(a, x_{j}\right)\right|}+\frac{\frac{1}{2}\left[a^{U}\left(x_{j}\right)-a^{L}\left(x_{j}\right)\right]}{\left|f\left(a, x_{i}\right) \cup f\left(a, x_{j}\right)\right|} ;$

(4) $a^{L}\left(x_{i}\right)<a^{L}\left(x_{j}\right)<a^{U}\left(x_{i}\right)<a^{U}\left(x_{j}\right)$, $p_{i j}^{a}=\frac{\frac{1}{2}\left[a^{U}\left(x_{i}\right)-a^{L}\left(x_{j}\right)\right]}{\left|f\left(a, x_{i}\right) \cup f\left(a, x_{j}\right)\right|} ;$

(5) $a^{L}\left(x_{j}\right)<a^{L}\left(x_{i}\right)<a^{U}\left(x_{i}\right)<a^{U}\left(x_{j}\right)$, $p_{i j}^{a}=\frac{\frac{1}{2}\left[a^{U}\left(x_{i}\right)-a^{L}\left(x_{i}\right)\right]}{\left|f\left(a, x_{i}\right) \cup f\left(a, x_{j}\right)\right|}+\frac{a^{L}\left(x_{i}\right)-a^{L}\left(x_{j}\right)}{\left|f\left(a, x_{i}\right) \cup f\left(a, x_{j}\right)\right|} ;$

(6) $a^{L}\left(x_{i}\right) \leq a^{U}\left(x_{i}\right) \leq a^{L}\left(x_{j}\right) \leq a^{U}\left(x_{j}\right)$, $p_{i j}^{a}=0$

Dominance relation

$$
R_{A}^{\geq}=\left\{\left(x_{i}, x_{j}\right) \in U \times U \mid p_{i j}>\frac{1}{2}, \text { or }_{i j}=\frac{1}{2}\left|f\left(a, x_{j}\right) \geq f\left(a, x_{i}\right)\right|\right\}
$$

$x_{i}$ of dominance is $\left[x_{i}\right]_{A}^{\geq}=\left\{x_{j} \in U \mid\left(x_{i}, x_{j}\right) \in R_{A}^{\geq}\right\}$.

Approximate size and roughness

Approximate size $\alpha_{R^{2}}(X)$ of Attribute $d$ and roughness is

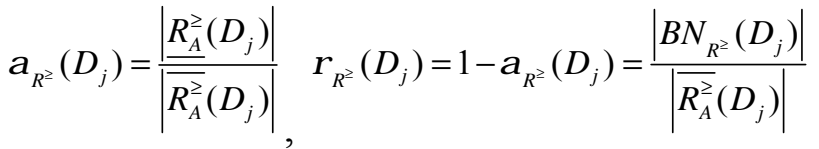

Conception 2.1 The rough entropy of subset $X$ is

$$
H_{R^{2}}(X)=-\frac{|X|}{|U|} \sum_{i=1}^{n} \frac{\left|\left[x_{i}\right]_{A T}^{2}\right| X \mid}{\left|\left[x_{i}\right]_{A T}^{2}\right|} \log \frac{\left|\left[x_{i}\right]_{A T}^{2}\right| X \mid}{\left|\left[x_{i}\right]_{A T}^{2}\right|}
$$

Within the multi-granularity approximation space, The uncertainty of acquiring knowledge is related to the breakdown of classification, The fine division, the smaller the uncertainty. 
Conception 3.1 $U=\left\{x_{1}, x_{2}, x_{3}, x_{4}, x_{5}\right\}, R_{1}^{\geq}, R_{2}^{\geq}$are two dominance relations, The calculation of dominant classes:

$$
\begin{aligned}
& U / R_{1}^{\geq}=\left\{[x i]_{R_{1}}^{\geq}: i=1, \ldots, 5\right\},\left[x_{1}\right]_{R_{1}}^{\geq}=\left\{x_{1}, x_{2}\right\},\left[x_{2}\right]_{R_{1}}^{\geq}=\left\{x_{2}\right\},\left[x_{3}\right]_{R_{1}}^{\geq}=\left\{x_{2}, x_{3}\right\}, \\
& {\left[x_{4}\right]_{R_{1}}^{\geq}=\left\{x_{1}, x_{2}, x_{4}\right\},\left[x_{3}\right]_{R_{1}}^{\geq}=\left\{x_{2}, x_{3}, x_{5}\right\},} \\
& U / R_{2}^{\geq}=\left\{[x i]_{R_{2}}^{\geq}: i=1, \ldots, 5\right\},\left[x_{1}\right]_{R_{2}}^{\geq}=\left\{x_{1}, x_{2}\right\},\left[x_{2}\right]_{R_{2}}^{\geq}=\left\{x_{2}\right\},\left[x_{3}\right]_{R_{2}}^{\geq}=\left\{x_{1}, x_{2}, x_{3}\right\}, \\
& {\left[x_{4}\right]_{R_{2}}^{\geq}=\left\{x_{1}, x_{2}, x_{3}, x_{4}\right\},\left[x_{3}\right]_{R_{2}}^{\geq}=\left\{x_{2}, x_{3}, x_{5}\right\},}
\end{aligned}
$$

There are the following properties:

Nature of 3.1 Rough entropy of $X$ meet $0 \leq H_{R^{2}}(X) \leq \frac{\left|X^{2}\right|}{|U|} \log \frac{|U|}{|X|}$.

Nature of 3.2 $X_{1}, X_{2} \subseteq U, H_{R^{2}}\left(X_{1} \cup X_{2}\right) \leq H_{R^{2}}\left(X_{1}\right)+H_{R^{2}}\left(X_{2}\right)$.

Exercises 1 An information system $I S=<U, A T, V, f>, \quad A T=\left\{a_{1}, a_{2}, a_{3}, a_{4}, a_{5}\right\}$, To solve the $\left.{ }^{H(D / C}\right)$

Table 1

\begin{tabular}{|r|c|r|r|r|r|r|}
\hline & $a_{1}$ & $a_{2}$ & $a_{3}$ & $a_{4}$ & $a_{5}$ & $d$ \\
\hline$x_{1}$ & {$[3,6]$} & {$[2,4]$} & {$[6,8]$} & {$[4,7]$} & {$[5,8]$} & 2 \\
\hline$x_{2}$ & {$[4,6]$} & {$[2,3]$} & {$[5,6]$} & {$[5,7]$} & {$[4,7]$} & 3 \\
\hline$x_{3}$ & {$[3,5]$} & {$[2,3]$} & {$[5,7]$} & {$[2,4]$} & {$[5,6]$} & 1 \\
\hline$x_{4}$ & {$[4,7]$} & {$[1,3]$} & {$[3,5]$} & {$[2,3]$} & {$[6,7]$} & 2 \\
\hline$x_{5}$ & {$[5,7]$} & {$[2,5]$} & {$[6,9]$} & {$[4,8]$} & {$[6,8]$} & 3 \\
\hline
\end{tabular}

$\left[x_{1}\right]_{A T}^{\geq}=\left\{x_{1}, x_{5}\right\},\left[x_{2}\right]_{A T}^{\geq}=\left\{x_{2}\right\},\left[x_{3}\right]_{A T}^{\geq}=\left\{x_{1}, x_{3}, x_{5}\right\},\left[x_{4}\right]_{A T}^{\geq}=\left\{x_{4}, x_{5}\right\},\left[x_{5}\right]_{A T}^{\geq}=\left\{x_{5}\right\}$

Decision attribute set $\{d\}$ divided into

$D_{1}=\left\{x_{3}\right\}, D_{2}=\left\{x_{1}, x_{4}\right\}, D_{3}=\left\{x_{5}\right\}$

According to the formula

$$
\begin{aligned}
& H_{R^{2}}\left(D_{1}\right)=\frac{1}{15} \log 3 H_{R^{2}}\left(D_{2}\right)=\frac{2}{5}\left(\log 2+\frac{1}{3} \log 3\right) H_{R^{2}}\left(D_{3}\right)=\frac{2}{5}\left(\log 2+\frac{1}{3} \log 3\right) \\
& H(D / C)=H_{R^{2}}\left(D_{1}\right)+H_{R^{2}}\left(D_{2}\right)+H_{R^{2}}\left(D_{3}\right)=\frac{1}{3} \log 3+\frac{4}{5} \log 2
\end{aligned}
$$

\section{Conclusions}

In this paper, the properties of rough set model are described by using the advantages relation, and the properties of the rough set model are presented by the rough entropy, and the example is used to analyze the model.

\section{Acknowledgements}

This work was financially supported by Guangxi university scientific research project 
(LX2014585).

\section{References}

[1]Pawlak.Z.Rough sets [J]. International Journal of Computer Information Sciences. 1982, 11(5): 342-356.

[2]Yuhua Qian,Jiye Liang. Positive approximation and rule extracting in incomplete information systems, Inter-national Journal of Computer Science and Knowledge Engineering, 2008, 2(1):51-63.

[3]W.H.Xu, X.Y.Zhang. Lower approximation reductions in inconsistent information systems based on dominance reductions. Computer engineering and applications. 2009, 45 (16) pp: 66-68.

[4]Junjun mao,Xia li .The relative entropy optimization model of attribute weighting based on rough set advantage relation is presented.[J]. The Computer engineering, 2011, 37(15):125-127.

[5] Yongjian zhai, Hong zhang. The advantages of incomplete information systems are more granular and coares-grained[J].Jounal of nanjing university of technology, 2012,36 (1) : 66-72.

[6]Qifang li, Jinjin li, Guoping li. The mufti-granularity rough set in the advantage relationship $[\mathrm{J}]$. Computer engineering and application, 2012,48 (17):53-57. 